\title{
How to calculate $\alpha$-decay rates in the future?
}

\author{
B. Gillis Carlsson ${ }^{\mathrm{a}}$, Daniel E. Ward, and Sven Åberg \\ Division of Mathematical Physics, LTH, Lund University, 22100 Lund, Sweden
}

\begin{abstract}
New elements discovered during past decades have been created in fusion reactions where a lighter nucleus is collided with a heavier one. The new elements created often decay by emitting $\alpha$ particles. From the half-lives of the decays and the energies of the emitted particles one may extract some properties of the new elements. In this talk the recent work performed by the Lund group to model $\alpha$ decay starting from nuclear density-functional theory is reviewed and a possible extension is mentioned.
\end{abstract}

\section{Introduction}

From theoretical predictions one expects around 7000 [1] different nuclear isotopes to exist. The current experimental tabulations contain around 2350 of these nuclei [2]. Out of all these different isotopes only around 300 are predicted to have half-lives longer than the expected life of the Solar System [1]. The remaining ones decay in various ways until they finally end up as stable isotopes. Many of the nuclei heavier than lead decay by sending out $\alpha$ particles. An emitted $\alpha$ particle travels with around 5\% of the speed of light. Due to collisions with the molecules in the air the particle typically only travels a few centimeters before it attracts electrons and combines with them to form an $\mathrm{He}$ atom. It is through this process that Helium gas is produced in the bedrock.

The new superheavy elements recently discovered also decay by emitting $\alpha$ particles. Typically the experimental groups can measure the average time before the created nucleus sends one out and its kinetic energy. Since energy is conserved in the process the measured kinetic energy tells us about the difference in mass between the initial nucleus and the resulting decay product. Sometimes the decay is repeated in several steps until one finally reaches a more long-lived isotope. The extracted mass differences obtained in each step allows us to connect the unknown mass of the new element to the mass of a known element. The mass, which is a complex quantity that cannot be calculated directly from the underlying theory of Quantum Chromo Dynamics (QCD), tells us how bound the nuclei are.

The time it takes for the decay to occur is even more complicated to calculate. The initial nucleus is created in a non-stationary state and the wave function will therefore evolve in time and explore the available phase space. After some time there is a chance that the initial nucleus is divided into a lighter nucleus and an $\alpha$ particle.

One way to model this process is to reduce the degrees of freedom of the system. Typically this is done by assuming that the system is composed of protons and neutrons treated as point particles. The interaction between these particles has been carefully deduced from scattering experiments. In this way ab-initio calculations can be performed for lighter elements but for

\footnotetext{
${ }^{\text {a }}$ Corresponding author: gillis.carlsson@matfys.lth.se
} 
heavy nuclei such calculations become intractable due to the complexity associated with the many interacting particles.

A method that enables calculations for the entire chart of nuclei and that can be extended to deal with descriptions of nuclear reactions is density-functional theory (DFT). During recent years the field of nuclear DFT has seen an amazing progress and in this talk we present the results of merging DFT models with a time-dependent description to obtain predictions for the half-lives of the heavy elements.

\section{Predictions of $Q_{\alpha}$ values}

The liquid drop model was originally proposed by Niels Bohr in 1936 and was later used by Bohr and Wheeler in 1939 [3] to explain nuclear fission. In this model, the probability for fission becomes large for heavy nuclei due to the repulsive effect of the Coulomb interaction. For nuclei with more than roughly 110 protons the fission barrier vanished and they were thus expected to be unstable and short lived. In more refined models, quantum mechanical effects due to the shell structure are predicted to have a stabilizing effect, enhancing the half-lives and thus opening the possibility for the existence of superheavy nuclei. In recent years the advancement of experimental methods have allowed the first exploration of this part of the nuclear chart.

The created superheavy nuclei often decay by sending out $\alpha$ particles. By summing the kinetic energy of the $\alpha$ particle and the recoil energy of the nucleus one obtains the $Q_{\alpha}$ value that specifies how much energy is released in the reaction. For nuclei with odd particle numbers the reaction often leaves the daughter nucleus in an excited state that may then further cool down by emitting photons. Summing the $Q_{\alpha}$ energy and the energy of the emitted photons one obtains the $Q_{\alpha}(g s)$ defined as the difference in ground-state binding energy before and after the reaction.

The measured $Q_{\alpha}(g s)$ values for the superheavy region of nuclei are illustrated with filled circles in Fig. 1. They should be compared with the full lines that show calculations based on the Skyrme-Hartree-Fock-Bogoliubov (SHFB) method using the SLy4 parametrization and a pairing functional of mixed type $[4,5]$. As seen in this figure the $Q_{\alpha}(g s)$ values are expected to decrease as one approaches the region around 180 neutrons. As the $Q_{\alpha}$ values become lower the half-lives increase and can lead to some very long-lived isotopes. Isotopes with both even and odd neutron numbers are shown in Fig. 1. Calculations for the isotopes with odd neutron numbers have also recently been carried out based on a Woods-Saxon potential [6] and a comparison between different models is presented in [7]. Most models give similar results for the $Q_{\alpha}(g s)$ values but can have important differences in details, especially as one approaches the spherical region around $N=184$ where collective correlations are expected to play a role.

For the ${ }_{98} \mathrm{Cf},{ }_{100} \mathrm{Fm},{ }_{102} \mathrm{No}$ and ${ }_{104} \mathrm{Rf}$ chains it has been possible to measure $\alpha$ decay to several different final states. The most common scenario is that the nucleus prefers to keep the odd neutron in the same orbital before and after the decay. The emitted $\alpha$ particle is thus formed by nucleons occupying a set of double-degenerate time-reversed orbitals. This type of decay is labeled favoured decay. The corresponding released energy $Q_{f}$ is shown in Fig. 1 with open symbols. In most of the situations this decay leaves the nucleus in an excited state that further deexcites through internal conversion or emission of $\gamma$ radiation. It is of interest to know when this will happen since the $\gamma$ radiation gives information of the level structure and the internal conversion process can be used to identify the elements. Whether or not the nucleus will decay through the favoured channel depends on a balance between the difficulty of forming the $\alpha$ particle and the difficulty for the $\alpha$ particle of escaping the nucleus. 


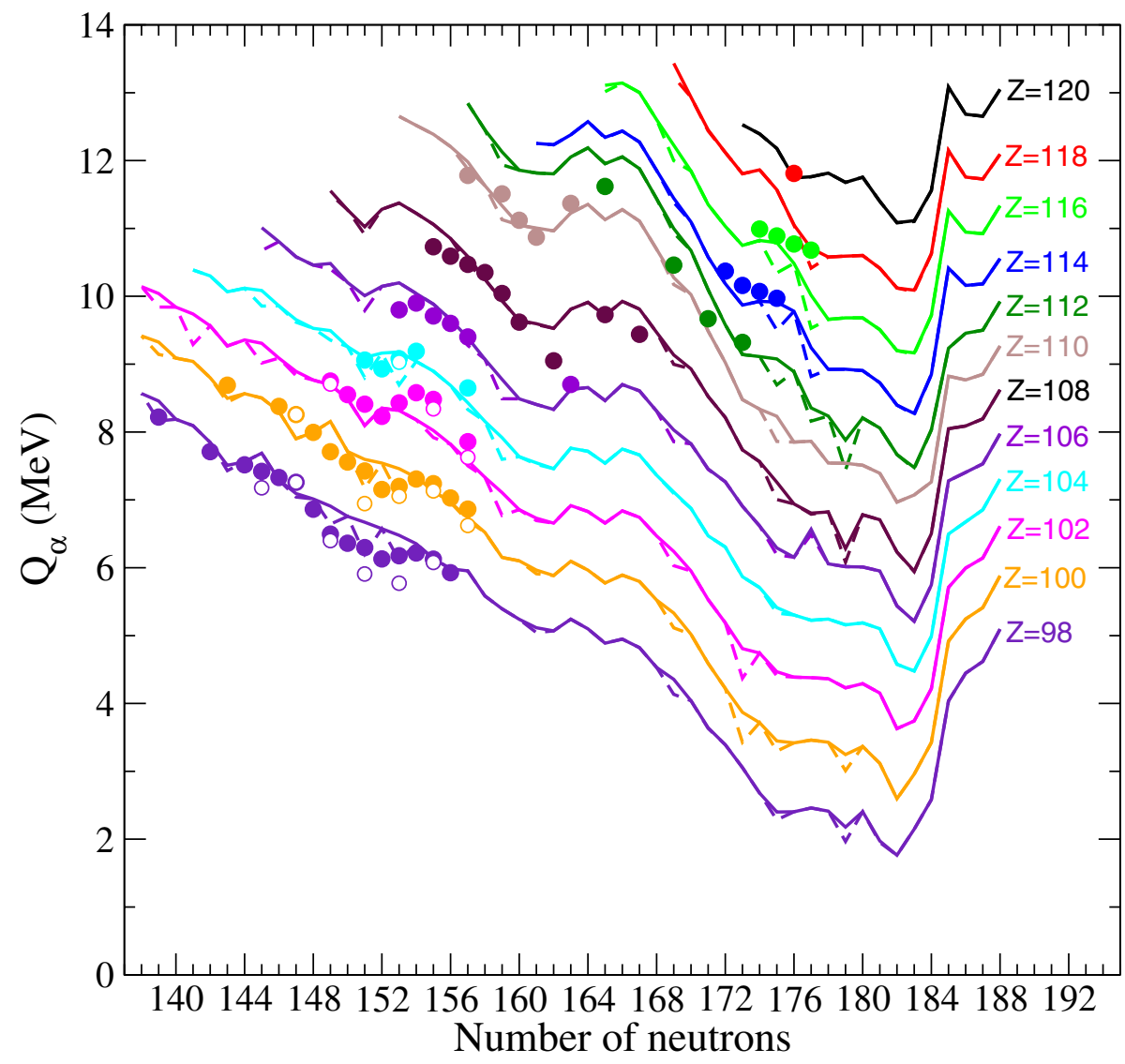

Figure 1. $Q_{\alpha}$ values of heavy and superheavy nuclei calculated using the SHFB model. Nuclei with odd particle numbers are calculated taking into account polarization effects in the equal-filling approximation [4]. The experimental data is taken from Refs. [6, 8].

The chance of formation increases for favoured decays but the chance of escaping often decreases due to a lowering of the $Q_{\alpha}$ value.

For illustrative purposes we may assume that if picking the favoured decay channel does not decrease the $Q_{\alpha}$ value by more than $0.5 \mathrm{MeV}$ then it decays through the favoured channel. The dashed lines in Fig. 1 illustrate the $Q_{f}$ value in the cases where $Q_{\alpha}(g s)-Q_{f}<0.5$. Along each isotope sequence one finds several cases where the odd nucleus is predicted to decay through the favoured channel enabling the observation of additional radiation. Taking the ${ }_{98} \mathrm{Cf}$ chain as an example the isotopes with $N=147,149,151$, and 153 have predicted favoured decays that are indeed observed, and the spins and parities of the levels are also predicted correctly. The change in energy from choosing the favoured decay compared to the ground-state decay is also predicted roughly correct. In the case of $N=145$ and 155 the model predicts the favoured decays to be the ground-state decays while in experiment the favoured decays lead to excited states in the daughter.

In general for the odd nuclei there is a competition between several decay channels populating different excited states in the daughter nuclei. To correctly model the decay scenario requires calculating the decay probabilities that depend on both the energies of the excited states as well as the structure of the mother and daughter wave functions. 


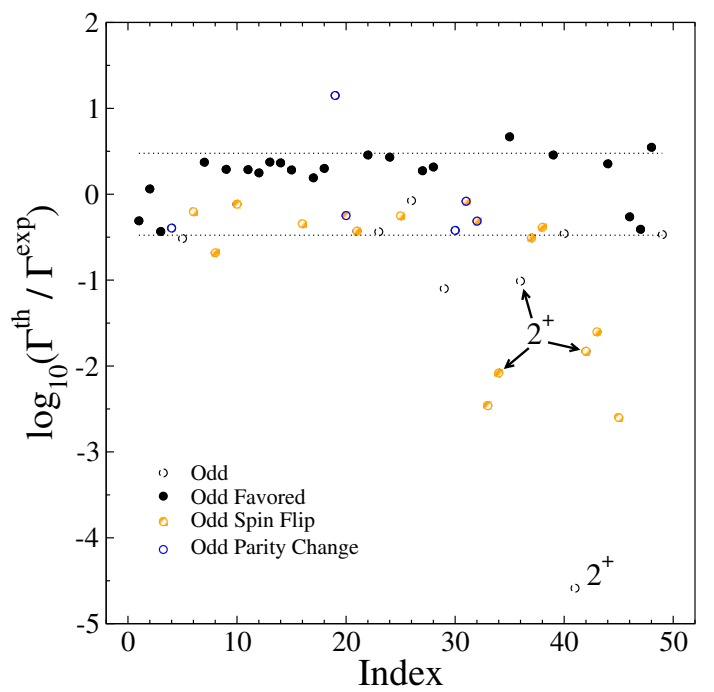

Figure 2. Logarithm of the ratio of the theoretical decay width to the experimental value for $\alpha$-decaying odd-A nuclei. The Skyrme parametrization SLy4 combined with mixed pairing is used in the calculations. The dotted lines indicate a deviation from experiment by a factor of three.

\subsection{Hindrance factors}

Determining the $\alpha$-decay path requires calculating the probability of decay to different excited states in the daughter nucleus. Such probabilities can be calculated by combining the SHFB model with a formalism to treat the $\alpha$-emission process. A microscopic description of the emission is obtained from the $R$-matrix approach [9]. The combination of these two frameworks was recently explored for $\alpha$ decay of spherical even-even [10] and odd nuclei [5]. The results show that within this formalism the nuclei are predicted to be much too long lived. A suggested explanation comes from missing many-body correlations that are not captured in the nuclear wave functions. Since relative decay rates were well reproduced the method could however be renormalized by a single constant. This implies that the missing correlation effects always increase the probability for the $\alpha$ particle to form with the same percentage. This assumption was tested by comparing calculations for all even-even [10] and odd spherical nuclei with experimental data [5].

Figure 2 shows the ratio of theoretical and experimental partial decay widths $\left(\Gamma=\hbar \ln (2) / t_{1 / 2}\right)$. The theoretical widths are calculated by combining the SHFB approach with the $R$-matrix approach [5]. All the data for spherical nuclei with odd particle numbers are included in the picture [5]. As seen from the figure most of the data can be reproduced with high accuracy. In a few cases the calculated widths were much smaller than the experimental values. Some of these points had previously been classified as having an odd particle in the daughter state coupled to a $2^{+}$vibration of the core. For such states our basic assumption of a pure one-quasiparticle excitation is violated. A description of these states could be obtained from a particle-vibration coupling model but this is outside the scope of our present investigation.

The decays can be classified according to whether the odd particle stays in the same orbital (favoured), changes direction of the intrinsic spin (spin flip), changes parity (parity change), or changes orbital but keeps spin and parity the same (orbital change). From the experimental data we can extract the hindrance factors for the decays belonging to the 
Table 1. Experimental hindrance factors for different groups of $\alpha$ decays extracted from spherical or close to spherical nuclei [5]. "No." denotes the number of data points in each group.

\begin{tabular}{llcc}
\hline Group & No. & $\begin{array}{c}\text { Geometric } \\
\text { Mean }\end{array}$ & $\begin{array}{c}\text { Geometric } \\
\text { Standard Deviation }\end{array}$ \\
\hline Even-even & 48 & 1 & 2.74 \\
Favoured odd & 22 & 2.57 & 2.97 \\
Spin flip & 14 & 163 & 3.22 \\
Parity change & 6 & 462 & 7.19 \\
Orbital change & 6 & 33.2 & 5.42 \\
All unfavoured odd & 23 & 117 & 5.44 \\
\hline
\end{tabular}

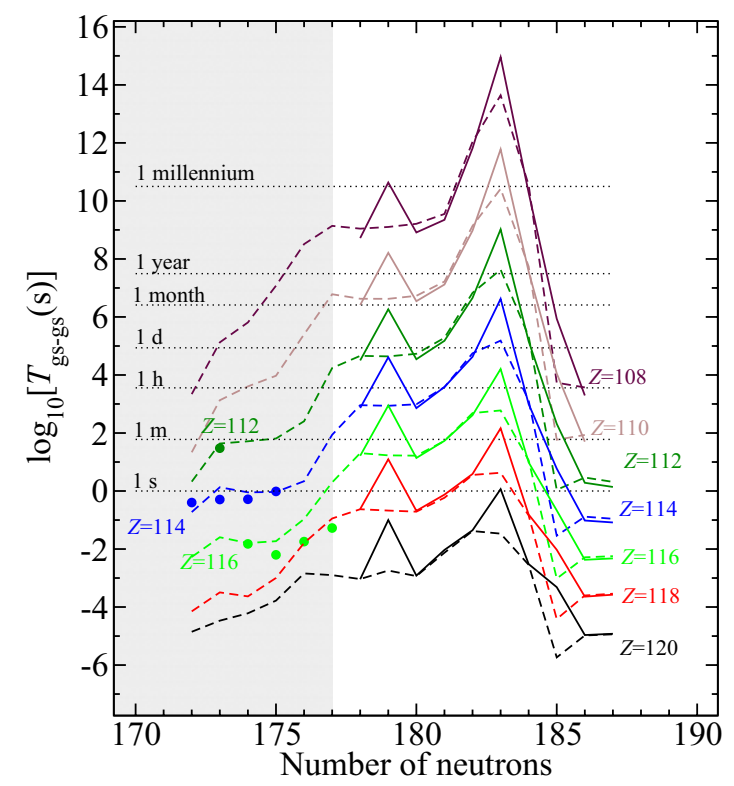

Figure 3. Alpha-decay half-lives of heavy and superheavy nuclei calculated using the SHFB model. The shaded area marks where the mother nuclei ground states are well deformed $|\beta|>0.1$.

different groups. Table 1 shows the hindrance factors extracted from experiment for the different types of decay. For even-even nuclei the average hindrance factor is taken to be 1 . For the favoured decays of odd nuclei they are on average hindered by a factor 2.57 meaning that they are roughly 2.57 times more long lived than an even-even decay having the same $Q_{\alpha}$ value. The geometric standard deviations for the different groups are quite large. For the favoured decays the half-lives vary with a factor 2.97 which is mainly due to the fluctuations in the pairing obtained when crossing shell gaps. Such fluctuations are not captured with the simple rules of thumb. Taking all the unfavoured decays as a single group the variation is a factor of 5.44 around an average hindrance of 117. This can be compared to the results in Fig. 2 where most of the decays fall within the dotted lines implying a difference between theory and experiment that is less than a factor three.

\subsection{Lifetimes of superheavy nuclei}

Figure 3 shows the calculated ground-state-to-ground-state $\alpha$-decay half-lives of the eveneven and odd nuclei in the superheavy island. The calculation is based on the SHFB model using the SLy4 parametrization and the predicted $Q_{\alpha}(g s)$ values shown in Fig. 1. 
The dashed lines corresponds to half-lives calculated without taking into account the hindrance due to differing wave functions. The solid lines show calculations where the structure of the mother and daughter nuclei are considered [5]. As seen in the figure one expects increased half-lives for ground-state-to-ground-state decays for $N=179$ corresponding to a hindered decay where the odd particle changes from a $d_{3 / 2}$ orbital to a $d_{5 / 2}$ orbital. The hindrance may cause the decay to instead follow the favoured path providing the difference in $Q_{\alpha}$ values are not too large. This competition was analyzed for the case of ${ }_{116} \mathrm{Lv}_{179}$ [5] where it was predicted that the decay will mainly go to the ground state with the odd particle moving from $d_{3 / 2}$ to $d_{5 / 2}$ resulting in an enhanced half-live for this isotope.

In general there are two main scenarios that will be the situation for many of the odd nuclei:

1. The main decay path is of unfavoured type and leaves the daughter in the ground state. In this case we expect enhanced half-lives as illustrated in Fig. 3. These half-lives will show a dependence on the orbitals involved.

2. The decay mainly follows the favoured channel leaving the daughter in an excited state. The measured $Q_{f}$ values will then tend to be smaller than for the neighbouring eveneven nuclei. In this case one further expects the nucleus to deexcite through $\gamma$ radiation or internal conversion that can give valuable information concerning the structure.

\section{Conclusions and outlook}

In conclusion, a short summary of our recent progress in the study of $\alpha$ decay of superheavy nuclei has been presented. The microscopic modeling of $\alpha$ decay is a challenge for theory. The approach discussed was able to give an improved description of hindrance factors that are important for predicting decay and half-lives of superheavy elements. Future experiments may utilize reactions such as titanium on berkelium that could lead to the creation of element 119 . The $\alpha$-decay chains will then go through the deformed region and are likely to lead to additional nuclear radiation being emitted. To simulate the decay chains the present formalism can be extended to treat deformed nuclear shapes. A combined theory and experimental study in this direction could lead to deeper insight into the structure of the nuclei around the superheavy island.

\section{References}

[1] J. Erler et al., Nature 486, 509 (2012).

[2] G. Audi et al., Chin. Phys. C 36, 1287 (2012).

[3] N. Bohr and J.A. Wheeler, Phys. Rev. 56, 426 (1939).

[4] M.V. Stoitsov et al., Comp. Phys. Comm. 184, 1592 (2013).

[5] D.E. Ward, B.G. Carlsson, S. Åberg, Phys. Rev. C 92, 014314 (2015).

[6] P. Jachimowicz, M. Kowal, J. Skalski, Phys. Rev. C 89, 024304 (2014).

[7] P.-H. Heenen et al., Nucl. Phys. A944, 415 (2015).

[8] The ENSDF database, http://www.nndc.bnl.gov/ensdf / (2016).

[9] R.G. Lovas et al., Phys. Rep. 294, 265 (1998).

[10] D.E. Ward, B.G. Carlsson, S. Åberg, Phys. Rev. C 88, 064316 (2013). 